\title{
Guest Editor's Preface: ULIA-2 Special Issue
}

As early as 1997, we decided to promote a short series of conferences devoted to a field that was showing dramatic progress and in which an increasing number of young researchers were participating. The Euroconference format of the "TMR Programme of the Commission of the European Union" was the most appropriate to fulfill the two objectives of a quasi real-time presentation and discussion of novel results and a tutorial support of young scientists from eminent lecturers active in the field. The success of the two meetings, held in two authentic capitals of the European cultural heritage, went well beyond any optimistic expectation. More than 200 enthusiastic participants, including approximately 100 young scientists, made those events a unique experience of scientific progress and debate. I wish to express my gratitude to Dimitris Charalambidis, who divided with me this experience, to the Scientific Advisory Committee, who guaranteed the high scientific level of the meetings, and to the Local Organizers, who made all this possible. The ULIA participants strongly appreciated the initiative of $\mathrm{La}$ ser and Particle Beams to edit two special issues with a collection of selected works presented at the ULIA meetings. Antonio Giulietti was the Scientific Promoter of the ULIA Conferences

The second Conference of the ULIA series took place in Pisa, Italy, from September 29 to October 3, 2000. The event, mainly supported by the European Commission and hosted by the Istituto di Fisica Atomica e Molecolare, was chaired by Dimitris Charalambidis from the University of Crete and the FORTH Foundation, Greece and co-chaired by Antonio Giulietti. The scientific sessions took place in the conference facility of the CNR Research Area of Pisa, located not far from the Leaning Tower and from the ancient walls of the old beautiful town. The Conference, introduced by the local authorities and by Professor Peter Mulser for the Scientific Advisory Committee, was opened by Professor Orazio Svelto with a lecture on Intense sub-10-femtosecond laser pulses: New physics on a table top.

Some 130 participants came from EU countries and from many other countries including Russia, Czech Republic, USA, Canada, Japan, Iran, and Israel. As for the first edition, held in Crete in 1999, almost one half of the participants were selected young scientists who were fully supported for participation to the event. They could benefit from high- level lectures delivered by leading scientists in the field including: F. Amiranoff, S.V. Bulanov, B. Cros, L. De Mauro, R. Doerner, O. Faucher, B. Girard, P. Lambropoulos, W. Leemans, D. von der Linde, A. Pukhov, C.K. Rhodes, C. Spielman, K. Taylor, J. Wark, and O. Willi. In addition, more than 30 oral papers and more than 70 poster contributions were presented in the 10 sessions of the event.

With no doubt, theoretical and experimental research on particle acceleration by ultrashort, ultraintense laser pulses in plasmas was the leading topic of the ULIA-2 Conference. Oral and poster sessions on this topic gave a clear view of the substantial progress both in experimental and theoretical research that has occurred in this field in the almost two years between the Crete and the Pisa events. A number of invited lectures were also devoted to particle acceleration mechanisms and the perspectives of their efficiency. Great attention was devoted also to both numerical simulations with highly sophisticated codes and complex experimental schemes tested in the leading laser facility worldwide. Further, a very interesting Panel Discussion, organized and chaired by Danilo Giulietti, which involved laser experts, plasma physicists, and high-energy physicists, provided a unique opportunity to discuss the perspectives of laserplasma particle acceleration for future high-energy physics experiments.

Other areas of investigation covered by the ULIA-2 Conference include: atomic physics and nonlinear optics in the presence of ultraintense electromagnetic fields; high-order harmonic generation; coherent and noncoherent $\mathrm{X}$-ray sources and their multidisciplinary use; technological applications as material processing; plasma physic studies of interest to nuclear fusion; and nuclear reactions produced from laser exploded clusters.

The scientific discussion was very intense during the conference sessions and informal during the free time, part of which was devoted to a guided visit to the medieval town of Pisa and to sample the typical delicious food and wines of Tuscany.

Leonida A. Gizzi Marco Galimberti IFAM-CNR, Pisa, Italy Guest Editors 